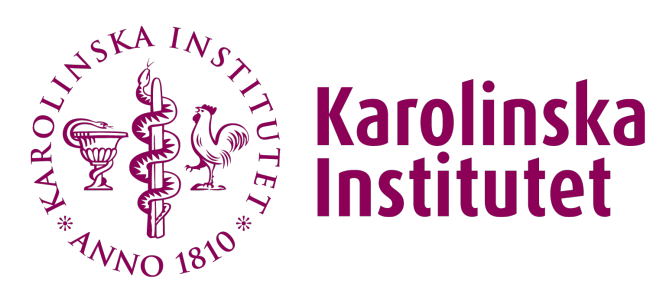

Karolinska Institutet

http://openarchive.ki.se

This is a Peer Reviewed Manuscript version of the following article, accepted for publication in The American Journal of Gastroenterology.

\title{
Association between metformin use and risk of esophageal squamous cell carcinoma in a population-based cohort study
}

Wang, Qiao-Li; Santoni, Giola; Ness-Jensen, Eivind; Lagergren, Jesper; Xie, Shao-Hua

Am J Gastroenterol. 2020 Jan;115(1):73-78.

Lippincott, Williams \& Wilkins

http://doi.org/10.14309/ajg.0000000000000478

http://hdl.handle.net/10616/47077

If not otherwise stated by the Publisher's Terms and conditions, the manuscript is deposited under the terms of the Creative Commons Attribution-NonCommercial-NoDerivatives License (http://creativecommons.org/licenses/by-nc-nd/4.0/), which permits non-commercial re-use, distribution, and reproduction in any medium, provided the original work is properly cited, and is not altered, transformed, or built upon in any way. 


\section{(1) \\ Karolinska \\ Institutet}

This is an author produced version of a paper accepted by American Journal of Gastroenterology. This paper has been peer-reviewed but does not include the final publisher proof-corrections or journal pagination.

Association between metformin use and risk of esophageal squamous cell carcinoma in a populationbased cohort study.

Wang, Qiao-Li; Santoni, Giola; Ness-Jensen, Eivind; Lagergren, Jesper; Xie, Shao-Hua.

Am J Gastroenterol. 2020 Jan;115(1):73-78.

DOI: https://doi.org/10.14309/ajg.0000000000000478

Access to the published version may require subscription.

Published with permission from: Wolters Kluwer Health, Inc. 
Title: Association between Metformin Use and Risk of Esophageal Squamous Cell Carcinoma in a Population-based Cohort Study

Running head: Metformin and Esophageal Squamous Cell Carcinoma Risk

\section{Authors:}

Qiao-Li Wang (MD) ${ }^{1}$, Giola Santoni $(\mathrm{PhD})^{1}$, Eivind Ness-Jensen (MD, PhD) ${ }^{1,2,3}$, Jesper Lagergren $(\mathrm{MD}, \mathrm{PhD})^{1,4}$, Shao-Hua Xie (MD, PhD) $)^{1}$

\section{Affiliations:}

${ }^{1}$ Upper Gastrointestinal Surgery, Department of Molecular medicine and Surgery, Karolinska Institutet, Karolinska University Hospital, Stockholm, Sweden.

${ }^{2}$ HUNT Research Centre, Department of Public Health and Nursing, NTNU, Norwegian University of Science and Technology, Levanger, Norway.

${ }^{3}$ Medical Department, Levanger Hospital, Nord-Trøndelag Hospital Trust, Levanger, Norway.

${ }^{4}$ School of Cancer and Pharmaceutical Sciences, King's College London, United Kingdom.

\section{Corresponding author:}

Dr Qiao-Li Wang, Upper Gastrointestinal Surgery, Department of Molecular medicine and Surgery, Karolinska Institutet, NS 67, 2nd Floor, Stockholm, 17176, Sweden, Tel.: +46 8-517 709 40, Fax: +46 8-517 762 80, E-mail: qiaoli.wang@ki.se

Word count: 2962 
Guarantor of the article: Senior authors Jesper Lagergren and Shao-Hua Xie take full responsibility for the conduct of the study and have had access to the data and have control of the decision to publish.

Specific author contributions: All authors have contributed to and approved the final version of the manuscript. The respective roles of each author are the following: study concept and design: all authors; data analysis: QLW, GS; interpretation of results: all authors; drafting the manuscript: QLW; critical revision of the manuscript for valuable intellectual content: all authors.

Financial support: This work was supported by the Swedish Research Council (521-2014-2536; 2015-06275, both Jesper Lagergren), the Swedish Cancer Society (CAN 2015/460, Jesper Lagergren), and the United European Research Prize (Jesper Lagergren). The study sponsors had no role in the study design, data collection, analysis, or interpretation, the writing of the report, or the decision to submit the manuscript for publication.

Potential competing interests: All authors declare no conflicts of interest.

Abbreviations: CI, confidence interval; ESCC, esophageal squamous cell carcinoma; HR, hazard ratio; ICD, International Classification of Diseases; ATC, Anatomical Therapeutic Chemical; NSAIDs, non-steroidal anti-inflammatory drugs; SD, standard deviation 


\section{STUDY HIGHLIGHTS}

\section{What is known}

- Esophageal squamous cell carcinoma (ESCC) is characterized by poor prognosis, highlighting a great need for chemo-prevention.

- The drug metformin may have anti-cancer properties.

- $\quad$ The impact of metformin use on ESCC is poorly investigated.

\section{What is new here}

- This nationwide population-based cohort study indicated a 32\% decreased risk of ESCC in metformin users compared to non-users.

- $\quad$ The decrease in risk remained in subgroup analyses and sensitivity analyses, indicating robustness of the finding. 


\section{ABSTRACT}

Objectives Esophageal cancer is a highly fatal malignant neoplasm, with two etiologically different histological types. A large prospective study is expected to elucidate the specific risk of the $90 \%$ subtype of esophageal cancer, esophageal squamous cell carcinoma, with metformin therapy. This study aims to determine the association between metformin use and incident esophageal squamous cell carcinoma risk.

Methods This was a nationwide population-based prospective cohort study conducted in Sweden in 2005-2015. Among 8.4 million participants identified in the cohort, 411,603 (5\%) were metformin users. The users were compared with 10 times as many frequency-matched non-users of metformin $(n=4,116,030)$ by age and sex. Metformin use was treated as a time-varying variate and multivariable cause-specific proportional hazards model was used to calculate hazard ratios (HR) with 95\% confidence intervals (CI) for esophageal squamous cell carcinoma, adjusted for age, sex, calendar year, residence area, tobacco smoking, alcohol overconsumption, and use of non-steroidal anti-inflammatory drugs or statins.

Results The incidence rates of esophageal squamous cell carcinoma were 3.5 per 100,000 personyears among the metformin users and 5.3 per 100,000 person-years in the non-users. Metformin users overall were at a decreased risk of esophageal squamous cell carcinoma compared with nonusers (HR 0.68, 95\% CI 0.54-0.85). The decrease in risk was more pronounced in new metformin users (HR 0.44, 95\% CI 0.28-0.64) and participants aged 60-69 years (HR 0.45, 95\% CI 0.310.66).

Conclusions Metformin use decreases the risk of developing esophageal squamous cell carcinoma.

Keywords Esophageal neoplasm; medication; risk factor; prevention 


\section{INTRODUCTION}

Esophageal cancer is the ninth most common cancer globally, with esophageal squamous cell carcinoma (ESCC) as the predominant (87\%) histological type (1, 2). Tobacco smoking and alcohol overconsumption explain more than 90\% of ESCC cases in Western populations (3-5). The prognosis of ESCC is poor with an overall five-year survival rate below 15-20\%, which highlights a great need for preventive measures (6).

Metformin is the most commonly used anti-diabetes drug, and it has a good safety profile and few side effects. Interestingly, a growing number of observational studies have suggested that the use of metformin decreases the risk of cancer overall and a few specific cancer types in particular, i.e., cancer of the colon or rectum, breast, and stomach (7-11). The only recently published randomized clinical trial found a decreased risk of tumorous progress in colorectal adenomas or polyps among metformin users (12). The limited existing literature examining metformin use in relation to the risk of esophageal cancer has provided conflicting results (13-16). Unfortunately, none of these studies has separated the two main histological types of esophageal cancer, i.e., squamous cell carcinoma and adenocarcinoma, despite the fact that these subtypes have very different aetiology (6). This study aimed to clarify the specific role of metformin in the development of ESCC. 


\section{METHODS}

\section{Study design and participants}

This was a population-based and nationwide Swedish cohort study carried out between July 1, 2005 to December 31, 2015. The study followed a priori-established protocol. The cohort was established within a well-defined database, entitled the Swedish Prescribed Drugs and Health (SPREDH) cohort. A detailed cohort profile for SPREDH has recently been published (17). Briefly, SPREDH includes 8.4 million ever-users of commonly prescribed medications, included from July 1, 2005, to December 31, 2015. The cohort participants and relevant data were identified from four nationwide health data registries in Sweden:

(1) Prescribed Drug Registry, established in 2005 (defining the start of this study) and containing data on all drugs prescribed and dispensed in outpatient care in the entire Swedish population;

(2) Patient Registry, covering $100 \%$ of inpatient care since 1987 and almost all specialized outpatient care from both public and private caregivers since 2001 in Sweden;

(3) Cancer Registry, established in 1958 with 96\% overall completeness for newly diagnosed cancers, and 98\% completeness specifically for esophageal cancer with $100 \%$ histological confirmation;

(4) Cause of Death Registry, established in 1952 with 100\% complete coverage for the date of death.

Information on drug use from the Prescribed Drug Registry included Anatomical Therapeutic Chemical (ATC) codes, defined daily doses (DDD), and dates of dispensing. This study was approved by the Regional Ethical Review Board in Stockholm (diary number 2016/4:6). 
The study population of the present study included participants in SPREDH who were older than 18 years and without any previous cancer diagnosis (except for non-melanoma skin cancer). Individuals who had used metformin before the age of 18 years were excluded. A flowchart of participants' enrolment is shown in online supplementary Figure 1.

The exposure was defined as having ever dispensed metformin during the study period. The exposed participants entered the study on the date of their first dispensation of metformin prescription. For each exposed participant (metformin user), ten comparison individuals in the cohort were randomly selected after frequency-matching by age (year of birth) and sex among those who had never used metformin at the time when the exposed person was identified. Thus, comparison individuals could become metformin users later. In addition to the total cohort examining all metformin users, a sub-cohort containing new (incident) metformin users and their matched comparison individuals was established from the original cohort. New users were defined as those without any metformin dispensation between July 1, 2005, and June 30, 2006 (one year's observation window, the maximal validation period for one prescription in Sweden), who had their first prescription dispensed after this period. To reduce potential detection bias, participants who developed ESCC within one year after entering the sub-cohort were excluded.

All participants were followed up until any of the following endpoints: diagnosis of ESCC, death, first metformin dispensation (non-exposed participants only) or the end of the study (December 31, 2015), whichever occurred first. The diagnosis of ESCC was determined in the Swedish Cancer Registry using a combination of the disease code C15 (tumor of the esophagus) in the $10^{\text {th }}$ version 
of the International Classification of Diseases (ICD-10) and the histological codes 8050-8078 or 8083-8084 (squamous cell carcinoma) in the third version of ICD for Oncology (ICD-O-3).

\section{Statistical analysis}

The incidence rate of ESCC and the cumulative incidence competing risk curve with K-sample test were calculated. We considered deaths from any cause as a competing event for incident ESCC. The association between metformin use and ESCC risk was assessed by time-varying analysis, treating metformin use as a time-dependent covariate. Multivariable cause-specific proportional hazards model was applied with adjustment of covariates, providing hazard ratios (HR) with 95\% confidence intervals (CI). Time since study entry was measured as the time scale for this model. The proportionality assumption of the hazard was met using the scaled Schoenfeld residuals. Covariates included age at study entry (continuous), sex (men or women), calendar year of study entry (continuous), tobacco smoking (yes or no, defined by tobacco smoking-related diagnoses within ten years before an individual's study entry), alcohol overconsumption (yes or no, defined by diagnoses related to alcohol overconsumption within ten years before entry), place of residence (eastern/southern Sweden or northern Sweden), use of a non-steroidal anti-inflammatory drug (NSAID) or aspirin (yes or no, within the year after study entry), and use of a statin (yes or no, within the year after study entry). Details about the ICD codes and ATC codes to identify the exposure, outcome, and covariates of this study are provided in online supplementary Tables 1 and 2. Three models were used to assess the influence of covariates: (1) a crude model (model 1); (2) a model adjusted for age, sex, calendar year, place of residence, tobacco smoking, and alcohol overconsumption (model 2); and (3) a model with further adjustment for use of NSAIDs/aspirin and statins (model 3). 
Stratified analyses were conducted by age at cohort entry (18-59, 60-69, and $\geq 70$ years), sex (men and women), and calendar period (2005-2009 and 2010-2015). Regarding the adjustments for covariates, the complete-case analysis was conducted because missing data amounted to less than $1 \%$.

A dose-response analysis was performed among all metformin users according to the total DDD of metformin intake within one year after first metformin dispensation. Metformin users were categorised into three approximately equal-sized groups: low ( $<175$ DDD), medium (175-300 DDD), and high (>300 DDD) dosages. The starting time for this specific analysis was one year after the first metformin dispensation. $P$ for trend was calculated by treating the metformin dosage as a continuous variable based on category-specific median values.

The following sensitivity analyses were conducted to evaluate the robustness of the results: (1) Censoring participants who developed any cancer (except for non-melanoma skin cancer) before ESCC during the follow-up; (2) Excluding participants who were followed up for $<1$ year, regardless of exposure status and reasons for the end of follow-up. All models, except for the doseresponse analysis, have been analyzed with a clustered sandwich estimator clustered on the matching group of the subjects (18).

To explore any protective effect of unmeasured or residual confounding over parameter constellations (19), a rule-out approach was applied for which prevalence of metformin use in the source cohort of $9 \%$ and a prevalence of a confounder of $80 \%$ was assumed. Using values found 
in the literature $(3,20-22)$, odds ratios of metformin use for non-smoking and non-alcohol overconsumption, and the relative risk of ESCC for non-smoking and non-alcohol overconsumption were assumed. To evaluate adherence to metformin therapy, the percentage of adherent participants was calculated by dividing the number of participants who had metformin dispensed at least once each specific calendar year with the total number of users from the previous year (excluding those who met any of the study endpoints), plus new users during the current year.

All analyses were performed using the statistical software package STATA MP 15.0 (StataCorp LLC, TX), except for the cumulative incidence curve which was computed using $\mathrm{R}$ package cmprsk. 


\section{RESULTS}

\section{Participants}

The total cohort included 411,603 metformin users (exposed) and 4,116,030 matched control

participants (non-exposed). Characteristics of the participants are presented in Table 1. The mean age at entry was 59.0 (standard deviation [SD] 13.7) years, and 58.2\% of the participants were men. The distribution of age, sex, residence area, tobacco smoking, and alcohol overconsumption was similar in users and non-users of metformin, while the use of NSAIDs and statins was more common in metformin users. The mean follow-up was 6.0 (SD 3.4) years in the metformin group and 5.8 (SD 3.5) years in the non-exposed group.

The sub-cohort of only new metformin users included 264,641 participants, accounting for 64.3\% of the metformin users in the total cohort. Characteristics of the sub-cohort of new metformin users are presented in online supplementary Table 3.

\section{Metformin use and esophageal squamous cell carcinoma risk}

A total of 1,352 newly diagnosed cases of ESCC occurred during follow-up. The incidence rates of ESCC were 3.5 per 100,000 person-years among metformin users and 5.3 per 100,000 personyears among non-users (Table 1). Compared with non-users, metformin users had a lower cumulative incidence of ESCC ( $p<0.001$; Figure 1). Metformin use was associated with a decreased HR of ESCC (adjusted HR 0.67, 95\% CI 0.54-0.84, model 2) and the HR was similar after further adjustment for the use of NSAIDs/aspirin and statins (adjusted HR 0.68, 95\% CI 0.540.85, model 3) (Table 2). The HR was lower among new users of metformin (adjusted HR 0.46, 95\% CI 0.31-0.67, model 2, and 0.44, 95\% CI 0.28-0.64, model 3) (Table 2). 
Stratified analyses showed a risk reduction among metformin users in both women (adjusted HR 0.62, 95\%CI 0.39-0.97, model 3) and men (adjusted HR 0.70, 95\% CI 0.54-0.90, model 3), and in participants aged 60-69 years (adjusted HR 0.45, 95\% CI 0.31-0.66), but not in the younger or older age groups (Table 3). A decreased risk was found for both calendar periods (Table 3).

The dose-response analysis showed that compared with metformin users with the lowest dose (DDDs $<175$ ), the point estimates were lower in the intermediate dose group (HR 0.91, 95\% CI 0.51-1.63) and in the high dose group (HR 0.89, 95\% CI 0.49-1.61), but these were not statistically significant (online supplementary Table 4).

\section{Sensitivity analyses}

In analyses excluding participants with other cancer before ESCC (sensitivity analysis 1) or participants with less than one year of follow up (sensitivity analysis 2), the results remained unchanged (Table 2).

\section{Influence of unmeasured confounding}

Online supplementary Figure 2 shows the influence of unmeasured confounders on the observed relative risk for the association between metformin use and ESCC risk. Under the assumption that odds ratios of metformin use for non-smoking and non-alcohol overconsumption were 0.22 and 0.32, respectively, and the relative risk of ESCC for non-smoking and non-alcohol overconsumption were 0.76 and 1.05 , respectively, potential unmeasured confounding from smoking and alcohol overconsumption should not alter the observed relative risk to null. Moreover, 
in order for the upper bound of the confidence interval of 0.85 to cross 1.0, if, e.g., the odds ratio between metformin and confounder is 2.13 , then that confounder has to decrease the relative risk of ESCC to as low as 0.34 or lower.

\section{Adherence to metformin therapy}

The percentage of participants who were adherent to metformin therapy was approximately $80 \%$

each year during the follow-up years, indicating relatively high and stable drug adherence and validity of the study (online supplementary Figure 3). 


\section{CONCLUSIONS}

This study indicates a decreased risk of ESCC among metformin users compared to non-users of metformin. The inverse association remained after considering the influence of confounding factors, competing risk from death, detection bias, residual or unmeasured confounding, and adherence to metformin therapy.

This study is the first to examine metformin use in relation to the risk of ESCC. Strengths include the large sample size, population-based cohort design, use of high-quality data, and complete follow-up. Selection bias should have been negligible in this study because of the Swedish national registries with excellent completeness and validity. Exposure information was collected prospectively and continuously, which eliminated concerns that may arise from retrospective data collection. The matched cohort study design counteracted potential time bias, which is common in other types of observational studies. This study also has limitations. The observational design cannot rule out unmeasured confounding, e.g., social-economic status, family history of ESCC, or concurrent head and neck cancer, as well as dietary and other lifestyle factors. However, some of these factors, e.g., family history of ESCC or concurrent head and neck cancer, are not likely to be strongly associated with the exposure and would therefore not confound the associations found in the present study. Confounding was taken into account by matching for age, sex and calendar period and adjusting for several potential confounding factors. Moreover, a rule-out approach assessing the influence of unmeasured confounding showed that it was unlikely to account for the entire observed reduction in HR of ESCC. Misclassification of medication probably exists, because it was not possible to verify that the participants actually used the medication after purchase. However, the personal metformin prescription was strictly followed by doctors in the 
country and the metformin users has to purchase the medicine with their identification card, which should strongly increase the probability that they actually used it. Finally, although the study was large, some of the sub-group analyses had limited statistical power to verify weak associations.

Without specifying the histological subtypes, a limited number of studies have reported conflicting results regarding the association between metformin use and the risk of esophageal cancer (13-16, 23). A case-control study from Taiwan reported an increased risk (odds ratio 2.84, 95\% CI 0.998.18) (24), while decreased risks were instead found in cohort studies from Taiwan (HR 0.49, 95\% CI 0.35-0.68) (13), and the Netherlands (HR 0.90, 95\% CI 0.82-0.97) (14). Other studies reported no association between metformin use and esophageal cancer risk $(15,16,23)$. The major differences in the findings of the available studies could be explained by differences in the distribution of squamous cell carcinoma and adenocarcinoma (and other less common histological types of esophageal cancer), study design, study population, data collection, adjustment for confounders, exposure measurement, comparison groups, and follow-up. A major limitation shared by all previous studies was that squamous cell carcinoma and adenocarcinoma were combined, although these two have distinct aetiology (6). Importantly, obesity is a strong risk factor for esophageal adenocarcinoma and is also strongly associated with diabetes $(6,25)$. Failure to control for the confounding from obesity in these studies could have yielded biased results (26). This problem was avoided in the present study because obesity is not a risk factor for ESCC. Similarly, diabetes increases the risk of some cancer types, but not ESCC $(7,27)$.

The decreased risk of ESCC among metformin users in this study remained after a series of sensitivity analyses, indicating the robustness of the finding. However, no clear dose-response 
association was found. This might have been due to the limited power in each group or that only a low dose was required to achieve a protective effect. Duration of metformin use and risk of ESCC was not analyzed in this cohort study because of concerns for time-related bias and reverse causality. The decrease in risk of ESCC was seemingly more pronounced among new metformin users, who started with a low dose, which may indicate a benefit of low-dose medication. Another explanation is that single-use of metformin is more common among new metformin users, while longer-term users more often also use other anti-diabetes medications, e.g., sulfonylurea or insulin, which can increase the risk of ESCC (24). The stratified analyses by age showed decreased point estimates of HRs in all age groups, but the association was statistically significant only in the age group 60-69 years. The lack of statistically significant association in the younger age group $(\leq 59$ years) might be explained by the low incidence of ESCC in younger age groups in combination with the relatively short follow-up (on average six years). In the older age group ( $\geq 70$ years) higher competing risk from mortality in diabetes patients could explain the weaker association. Yet, because of the lower power in the stratified analyses, the possible differences in findings regarding new metformin users and the age groups should be interpreted with caution.

Biological mechanisms that may explain how metformin may counteract cancer development have been widely investigated. Metformin modulates hyperinsulinemia by interruption of insulin binding to the insulin receptor or the insulin-like growth factor-1 receptor. This leads to deactivation of the phosphoinositide 3-kinase pathway or mitogen-activated protein-kinase, reduction of protein synthesis, and cell proliferation (28). Metformin can also deplete energy in cells and activate the 5' adenosine monophosphate-activated protein kinase (AMPK) pathway, the cellular energy regulator center, and influence protein synthesis, lipogenesis, angiogenesis, cell 
cycle arrest, interruption of cancer stem cell differentiation, and promotion of anti-inflammation (8). Activation of AMPK by metformin is a necessary mediator of the tumor suppressor liver kinase B1 (LKB1), especially in patients with metabolic syndrome, e.g., diabetes (29). The anticancer properties of metformin may also be due to the promotion of autophagy and activation of the immune system (8). However, mechanisms specific for ESCC remain to be investigated.

The reduced risk of ESCC among metformin users identified in this study remains to be validated in independent populations. If the strongly decreased risk found in this study is supported by further research, it may lay the foundation for randomized clinical trials in high-risk populations of ESCC, e.g., in parts of France, China, and Japan (30).

In conclusion, this nationwide and population-based cohort study in Sweden indicates that metformin use decreases the risk of ESCC. 


\section{REFERENCES}

1. Bray F, Ferlay J, Soerjomataram I, et al. Global cancer statistics 2018: GLOBOCAN estimates of incidence and mortality worldwide for 36 cancers in 185 countries. CA Cancer J Clin 2018;68:394-424.

2. Arnold M, Soerjomataram I, Ferlay J, et al. Global incidence of oesophageal cancer by histological subtype in 2012. Gut 2015;64:381-7.

3. Wang QL, Xie SH, Li WT, et al. Smoking Cessation and Risk of Esophageal Cancer by Histological Type: Systematic Review and Meta-analysis. Jnci-Journal of the National Cancer Institute 2017;109.

4. Lagergren J. Influence of obesity on the risk of esophageal disorders. Nat Rev Gastroenterol Hepatol 2011;8:340-7.

5. Lagergren J, Bergstrom R, Lindgren A, et al. Symptomatic gastroesophageal reflux as a risk factor for esophageal adenocarcinoma. N Engl J Med 1999;340:825-31.

6. Lagergren J, Smyth E, Cunningham D, et al. Oesophageal cancer. Lancet 2017;390:2383-2396.

7. Giovannucci E, Harlan DM, Archer MC, et al. Diabetes and cancer: a consensus report. CA Cancer J Clin 2010;60:207-21.

8. Pawalowska M, Markowska A. The influence of metformin in the etiology of selected cancers. Contemp Oncol (Pozn) 2012;16:223-9.

9. Cheung KS, Chan EW, Wong AYS, et al. Metformin Use and Gastric Cancer Risk in Diabetic Patients After Helicobacter pylori Eradication. J Natl Cancer Inst 2018.

10. Zhang ZJ, Zheng ZJ, Kan H, et al. Reduced risk of colorectal cancer with metformin therapy in patients with type 2 diabetes: a meta-analysis. Diabetes Care 2011;34:2323-8.

11. Bodmer M, Meier C, Krahenbuhl S, et al. Long-term metformin use is associated with decreased risk of breast cancer. Diabetes Care 2010;33:1304-8.

12. Higurashi T, Hosono K, Takahashi $\mathrm{H}$, et al. Metformin for chemoprevention of metachronous colorectal adenoma or polyps in post-polypectomy patients without diabetes: a multicentre doubleblind, placebo-controlled, randomised phase 3 trial. Lancet Oncol 2016;17:475-483.

13. Tseng $\mathrm{CH}$. Metformin and esophageal cancer risk in Taiwanese patients with type 2 diabetes mellitus. Oncotarget 2017;8:18802-18810.

14. Ruiter R, Visser LE, van Herk-Sukel MP, et al. Lower risk of cancer in patients on metformin in comparison with those on sulfonylurea derivatives: results from a large population-based follow-up study. Diabetes Care 2012;35:119-24.

15. Becker C, Meier CR, Jick SS, et al. Case-control analysis on metformin and cancer of the esophagus. Cancer Causes Control 2013;24:1763-70.

16. Lee MS, Hsu CC, Wahlqvist ML, et al. Type 2 diabetes increases and metformin reduces total, colorectal, liver and pancreatic cancer incidences in Taiwanese: a representative population prospective cohort study of 800,000 individuals. BMC Cancer 2011;11:20.

17. Shao-Hua Xie GS, Fredrik Mattsson, Eivind Ness-Jensen, Jesper Lagergren. Cohort profile: The Swedish Prescribed Drugs and Health Cohort (SPREDH). 2018.

18. Lin DY, Wei LJ. The Robust Inference for the Cox Proportional Hazards Model. Journal of the American Statistical Association 1989;84:1074-1078.

19. Schneeweiss S. Sensitivity analysis and external adjustment for unmeasured confounders in epidemiologic database studies of therapeutics. Pharmacoepidemiol Drug Saf 2006;15:291-303.

20. Li XH, Yu FF, Zhou YH, et al. Association between alcohol consumption and the risk of incident type 2 diabetes: a systematic review and dose-response meta-analysis. Am J Clin Nutr 2016;103:818-29. 21. Islami F, Fedirko V, Tramacere I, et al. Alcohol drinking and esophageal squamous cell carcinoma with focus on light-drinkers and never-smokers: a systematic review and meta-analysis. Int J Cancer 2011;129:2473-84. 
22. Yeh HC, Duncan BB, Schmidt MI, et al. Smoking, smoking cessation, and risk for type 2 diabetes mellitus: a cohort study. Ann Intern Med 2010;152:10-7.

23. Murff HJ, Roumie CL, Greevy RA, et al. Metformin use and incidence cancer risk: evidence for a selective protective effect against liver cancer. Cancer Causes Control 2018;29:823-832.

24. Cheng KC, Chen YL, Lai SW, et al. Risk of esophagus cancer in diabetes mellitus: a populationbased case-control study in Taiwan. BMC Gastroenterol 2012;12:177.

25. Chatterjee S, Khunti K, Davies MJ. Type 2 diabetes. Lancet 2017;389:2239-2251.

26. Lagergren J, Bergstrom R, Nyren O. Association between body mass and adenocarcinoma of the esophagus and gastric cardia. Ann Intern Med 1999;130:883-90.

27. Inoue $\mathrm{M}$, Iwasaki $\mathrm{M}$, Otani $\mathrm{T}$, et al. Diabetes mellitus and the risk of cancer: results from a largescale population-based cohort study in Japan. Arch Intern Med 2006;166:1871-7.

28. Joo MK, Park JJ, Chun HJ. Additional Benefits of Routine Drugs on Gastrointestinal Cancer:

Statins, Metformin, and Proton Pump Inhibitors. Dig Dis 2018;36:1-14.

29. Luo Z, Zang M, Guo W. AMPK as a metabolic tumor suppressor: control of metabolism and cell growth. Future Oncol 2010;6:457-70.

30. Wang QL, Xie SH, Wahlin K, et al. Global time trends in the incidence of esophageal squamous cell carcinoma. Clin Epidemiol 2018;10:717-728. 


\begin{tabular}{|c|c|c|c|c|}
\hline \multicolumn{2}{|l|}{ Characteristic } & $\begin{array}{r}\text { All participants } \\
(\mathrm{n}=4,527,633) \\
\text { Number }(\%)\end{array}$ & $\begin{array}{r}\text { Metformin users } \\
(\mathrm{n}=411,603) \\
\text { Number }(\%) \\
\end{array}$ & 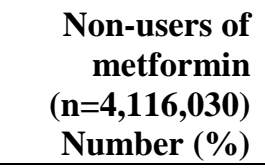 \\
\hline \multicolumn{2}{|c|}{ Age, mean years (SD) } & $59.0( \pm 13.7)$ & $59.0( \pm 13.7)$ & $59.0( \pm 13.7)$ \\
\hline \multicolumn{5}{|c|}{ Sex } \\
\hline & Men & $2,632,960(58.2)$ & $239,360(58.2)$ & 2,393,600 (58.2) \\
\hline & Women & $1,894,673(41.8)$ & $172,243(41.8)$ & $1,722,430(41.8)$ \\
\hline \multicolumn{5}{|c|}{ Residence in Sweden } \\
\hline & Eastern/Southern & 3,604,966 (79.6) & $320,963(78.0)$ & $3,284,003(79.8)$ \\
\hline & Northern & $913,051(20.2)$ & $90,074(21.9)$ & $822,977(20.0)$ \\
\hline & Unknown & $9,616(0.2)$ & $566(0.1)$ & $9,050(0.2)$ \\
\hline Smoking & & $115,347(2.5)$ & $11,794(2.9)$ & $103,553(2.5)$ \\
\hline \multicolumn{2}{|c|}{ Alcohol overconsumption ${ }^{\mathrm{a}}$} & $112,486(2.5)$ & $10,427(2.5)$ & $102,059(2.5)$ \\
\hline \multicolumn{2}{|c|}{ Use of NSAIDs or aspirin } & $1,666,716(36.8)$ & $200,182(48.6)$ & $1,466,534(35.6)$ \\
\hline \multicolumn{2}{|c|}{ Use of statins } & $1,041,094(23.0)$ & $212,815(51.7)$ & $828,279(20.1)$ \\
\hline \multicolumn{2}{|c|}{ Duration of follow-up, mean years (SD) } & $5.8( \pm 3.4)$ & $6.0( \pm 3.4)$ & $5.8( \pm 3.5)$ \\
\hline \multirow{2}{*}{\multicolumn{2}{|c|}{$\begin{array}{l}\text { ESCC cases } \\
\text { ESCC incidence per 100,000 person-years } \\
(95 \% \text { CI) }\end{array}$}} & $1,352(0.03)$ & $86(0.02)$ & $1,266(0.03)$ \\
\hline & & $5.1(4.8-5.4)$ & $3.5(2.8-4.3)$ & $5.3(5.0-5.6)$ \\
\hline \multicolumn{2}{|c|}{ Death during follow-up } & $598,899(13.2)$ & $71,409(17.4)$ & $527,490(12.8)$ \\
\hline \multicolumn{2}{|c|}{ New metformin users ${ }^{\mathrm{b}}$} & NA & $264,641(64.3)$ & NA \\
\hline
\end{tabular}


Table 2. Risk of esophageal squamous cell carcinoma (ESCC) in metformin users compared with matched non-users of metformin in Sweden in 2005-2015

\begin{tabular}{|c|c|c|c|c|}
\hline \multirow[b]{2}{*}{ Analysis } & \multirow[b]{2}{*}{ ESCC (number) } & \multicolumn{3}{|c|}{ Hazard ratio ( $95 \%$ confidence interval) } \\
\hline & & Model 1 & Model 2 & Model 3 \\
\hline Metformin use & 1,348 & $0.66(0.53-0.82)$ & $0.67(0.54-0.84)$ & $0.68(0.54-0.85)$ \\
\hline New metformin use & 627 & $0.46(0.32-0.67)$ & $0.46(0.31-0.67)$ & $0.44(0.28-0.64)$ \\
\hline Sensitivity analysis $1^{\mathrm{a}}$ & 1,210 & $0.68(0.54-0.85)$ & $0.69(0.55-0.87)$ & $0.70(0.56-0.88)$ \\
\hline Sensitivity analysis $2^{\mathrm{b}}$ & 1,142 & $0.66(0.52-0.84)$ & $0.67(0.53-0.85)$ & $0.68(0.53-0.86)$ \\
\hline
\end{tabular}

Model 1: crude analysis

Model 2: adjusted by age, sex, calendar year, residence, smoking, and alcohol overconsumption

Model 3: model 2 further adjusted by non-steroidal anti-inflammatory drugs/aspirin and statin use

${ }^{a}$ Censored those who developed any other cancers (except for non-melanoma skin cancer) before ESCC during follow-up

${ }^{\mathrm{b}}$ Excluded those with less than one-year follow-up, regardless of exposure status and reasons for the end of follow-up

Note: All results met the proportionality assumption of the hazard 
Table 3. Risk of esophageal squamous cell carcinoma (ESCC) in metformin users compared with matched nonusers of metformin in Sweden in 2005-2015, stratified by sex, age, or calendar period

\begin{tabular}{|c|c|c|}
\hline Category & ESCC (number) & $\begin{array}{r}\text { Hazard ratio } \\
(95 \% \text { confidence interval) }\end{array}$ \\
\hline Total & 1,348 & $0.68(0.54-0.85)$ \\
\hline \multicolumn{3}{|l|}{ Sex } \\
\hline Men & 972 & $0.70(0.54-0.90)$ \\
\hline Women & 376 & $0.62(0.39-0.97)$ \\
\hline \multicolumn{3}{|l|}{ Age, years } \\
\hline$\leq 59$ & 279 & $0.90(0.56-1.45)$ \\
\hline $60-69$ & 595 & $0.45(0.31-0.66)$ \\
\hline$\geq 70$ & 474 & $0.92(0.66-1.29)$ \\
\hline \multicolumn{3}{|l|}{ Calendar period } \\
\hline 2005-2009 & 1,094 & $0.67(0.52-0.86)$ \\
\hline $2010-2015$ & 254 & $0.69(0.41-1.16)$ \\
\hline
\end{tabular}

a Adjusted for age, sex, calendar year, residence, smoking, and alcohol overconsumption, non-steroidal anti-inflammatory drugs/aspirin and statin use, except for the evaluation variable.

Note: All results met the proportionality assumption of the hazard 


\section{FIGURE LEGENDS}

Figure 1: Cumulative incidence of patients developing esophageal squamous cell carcinoma (ESCC) during follow-up among metformin users and non- users of metformin. 


\section{LEGENDS OF SUPPLEMENTARY MATERIALS}

Supplementary Table 1: International Classification of Diseases (ICD) codes for outcomes and covariates

Supplementary Table 2: Anatomical Therapeutic Chemical (ATC) codes for exposure and covariates

Supplementary Table 3: Baseline characteristics of sub-cohort of new metformin users

Supplementary Table 4: Risk of esophageal squamous cell carcinoma (ESCC) in metformin users with dosage level during the first year (expressed as defined daily dose, DDD) in Sweden in 2005-2015.

Supplementary Figure 1: Flowchart of participants’ enrolment

Supplementary Figure 2: Influence of unmeasured confounders on the observed relative risk of metformin use and esophageal squamous cell carcinoma $X$-axis: relative risk $\left(R R_{C D}\right)$ of unmeasured confounder and esophageal squamous cell carcinoma (ESCC, outcome); Y-axis: odds ratio $\left(O R_{E C}\right)$ of metformin use (exposure) and unmeasured confounder. The solid line shows the association between $R R_{C D}$ and $O R_{E C}$ when the apparent (observed) relative risk (ARR) between metformin use (exposure) and ESCC (outcome) is 0.68 and when the true relative risk is 1 . The dashed line shows the association between $R R_{C D}$ and $O R_{E C}$ when the ARR between metformin use and ESCC is 0.85 and when the true relative risk is 1 . The analysis was under the assumption of a prevalence of the confounder of $80 \%$ and a prevalence of metformin use of $9 \%$. Any combination of $R R_{C D}$ and $O R_{E C}$ that lies in the area to the lower right of each line indicates the unmeasured confounders that would not move the ARR to the null.

Supplementary Figure 3: Percent of adherent to metformin therapy among metformin users during follow-up 